\title{
One-Step Cellular Caspase-3/7 Assay
}

BioTechniques 34:1064-1067 (May 2003)

Rosa A. Carrasco, Nancy B. Stamm, and Bharvin K.R. Patel

Eli Lilly and Company, Indianapolis, IN, USA

Apoptosis or programmed cell death is the carefully regulated process of cell death that plays important roles in a wide variety of normal processes, ranging from fetal development to immune system regulation (1-4). Morphologically, apoptosis is characterized by chromatin condensation, nuclear fragmentation, and the loss of the mitochondrial inner transmembrane potential, which results in plasma membrane blebbing, with cells breaking into small membrane-surrounded fragments (apoptotic bodies) that are cleared by phagocytosis (5). These morphological changes are believed to be caused by a family of cysteine proteases that are known as caspases. The recognition site for caspases is marked by three to four amino acids, followed by an aspartic acid residue, with cleavage occurring after the aspartate $(6,7)$. Caspases are typically synthesized as inactive zymogens but are activated as a result of their proteolytic processing at conserved internal aspartate through autocatalysis or by another protease $(7,8)$. Caspase- 3 is a key downstream effector in the apoptosis pathway that amplifies the signal from initiator caspases such as caspase- 8 and caspase-9 (9). Caspase-3 has been shown to cleave poly(ADP-ribose) polymerase (PARP), DNA-dependent protein kinase, PKC$\delta$, and actin (10).

Defects in the apoptotic pathway that cause hypo- or hyperactivity of apoptosis contribute significantly to multiple diseases such as cancer, Alzheimer's disease, Parkinson's disease, heart failure, restenosis, stroke, osteoporosis, AIDS, allograft rejection, autoimmune disorders, trauma, and infectious diseases. The importance of apoptosis in these diseases has generat- ed strong interest in devising therapeutic strategies for modulating apoptotic pathways $(11,12)$. The identification of compounds that inhibit apoptosis could potentially treat neurodegenerative diseases, or compounds capable of inducing apoptosis may be useful to treat cancer $(10,13)$. To identify such com-

\section{Protocol for Caspase-3/7 Assay}

1. Plate desired number of cells in $50 \mu \mathrm{L}$ media/well in a 96-well plate. Incubate the plate overnight at $37^{\circ} \mathrm{C}$ for the cells to adhere. We recommend optimizing the cell densities for each cell line under investigation for the 96-well or 384well plates.

2. The following day, add serially diluted compounds under investigation in a 50$\mu \mathrm{L}$ culture media. Incubate at $37^{\circ} \mathrm{C}$ for the required period of time.

3. After incubation, add $50 \mu \mathrm{L}$ of one-step caspase-3/7 assay buffer $(3 \times) /$ well and incubate at $37^{\circ} \mathrm{C}$ for $1 \mathrm{~h}$.

Caspase-3/7 Assay Buffer ( $3 \times$ ): Prepare the following buffer, filter, and store $5.64 \mathrm{~mL}$ (sufficient for one plate) aliquots at $-20^{\circ} \mathrm{C}$ to $-70^{\circ} \mathrm{C}$ until needed.

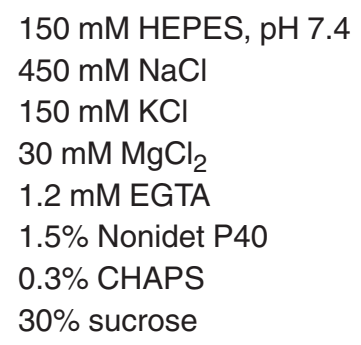

Use $50 \mu \mathrm{L} 3 \times$ buffer/well containing cells in $100 \mu \mathrm{L}$ culture medium.

4. Measure the caspase-3/7 activity by reading proteolytically released fluorochrome from the DEVD-AMC substrate using a plate-reading fluorometer with an excitation at $360 \mathrm{~nm}$ and an emission at $460 \mathrm{~nm}$.

5. Input these data into the GraphPad Prism software to calculate the $\mathrm{EC}_{50}$ (concentration required to induce caspase-3/7 activity in a given sample by $50 \%$ versus untreated control). 
pounds, one needs a high-throughput assay that is robust and relatively inexpensive. We have therefore developed a rapid cell-based caspase-3/7 assay that is very simple and amenable to high throughput to screen compounds that are capable of modulating apoptosis.

Traditionally, the measurement of cellular caspase-3/7 enzymatic activity requires a large number of cells (1-3 $\times$ $\left.10^{6}\right)$. The cells are plated in a $10-\mathrm{cm}$ Petri dish or a 6-well plate to adhere overnight and treated with apoptosismodulating compound for a desired length of time, followed by media withdrawal, washing with PBS, and collection of cellular protein in a lysis buffer (20 mM Tris-Cl, pH 7.4, 5 mM EDTA, $50 \mathrm{mM} \mathrm{NaCl}, 10 \mathrm{mM}$ sodium pyrophosphate, $50 \mathrm{mM}$ sodium fluoride, and $1 \%$ Nonidet $^{\mathrm{TM}} \mathrm{P} 40$ ). Protein concentration from each sample is measured by an appropriate method, and $50-150 \mu \mathrm{g}$ of total protein are used in an in vitro enzymatic reaction buffer containing N-acetyl-DEVD-7-amino4-methlycoumarin (DEVD-AMC) peptide (caspase-3/7 substrate) (BIOMOL Research Laboratories, Plymouth Meeting, PA, USA). After incubation, the enzymatic activity is measured by detecting cleaved substrate using a CytoFluor ${ }^{\circledR}$ series 4000 fluorescence plate reader (PerSeptive Biosystems, Framingham, MA, USA). Figure 1A shows that the treatment of HeLa cells with staurosporine for $4 \mathrm{~h}$ results in caspase-3/7 activation in a dose-dependent manner. To confirm the observed cellular caspase-3/7 activity, $75 \mu \mathrm{g}$ of total protein from each sample were electrophoretically separated on $4 \%$ $15 \%$ SDS-polyacrylamide gels, transferred to PVDF membrane, and probed with antibodies to detect caspase-3, caspase-9, PARP, and control protein actin. Increased caspase-3/7 enzymatic activity is confirmed by the reduction in the level of the inactive form of caspase-3 (the active form of caspase- 3 cannot be detected by immunoblotting), increasing the levels of active caspase-9, and the cleaved form of PARP (Figure 1B). It is clear that measuring cellular enzymatic activity is more sensitive than looking for PARP cleavage by immunoblotting, as evidenced by the detection of caspase- $3 / 7$ activity in HeLa cells treated with as little as $0.03125 \mu \mathrm{M}$ staurosporine versus $0.25 \mu \mathrm{M}$ by immunoblotting. Additionally, a very small number of samples can be processed using this rather laborious procedure that is unsuitable to screen thousands of compounds.

Therefore, we devised a rapid onestep assay to measure the cellular caspase-3/7 enzymatic activity (see Protocol for Caspase-3/7 Assay) in a 96-well format. This assay involves a one-step buffer containing components required for efficient cell lysis and 


\section{Benchmark}

reagents required for the enzymatic reaction. The assay is very rapid and requires no time for sample preparation. Cells that have been treated with apoptosis-modulating agents receive buffer containing substrates for caspase-3/7. The buffer is added directly to each well, and the plate is incubated at $37^{\circ} \mathrm{C}$ for $1 \mathrm{~h}$. After incubation, a simple fluorescence plate reader measures the released fluorochrome in each well. This one-step buffer offers a significant advantage because it does not require the removal of culture media and a washing step that could potentially remove apoptotic cells from wells, and it al- lows the assay to be performed on cells grown in suspension. The assay is amenable to automation for high throughput and secondary screening to identify active compounds that modulate apoptosis. The data are plugged into GraphPad Prism ${ }^{\circledR}$ software (GraphPad Software, San Diego, CA, USA) to calculate the $\mathrm{EC}_{50}$ (concentration required to induce caspase- $3 / 7$ activity in a given sample by $50 \%$ versus the untreated control). To demonstrate the robustness of this assay to screen

\section{A}

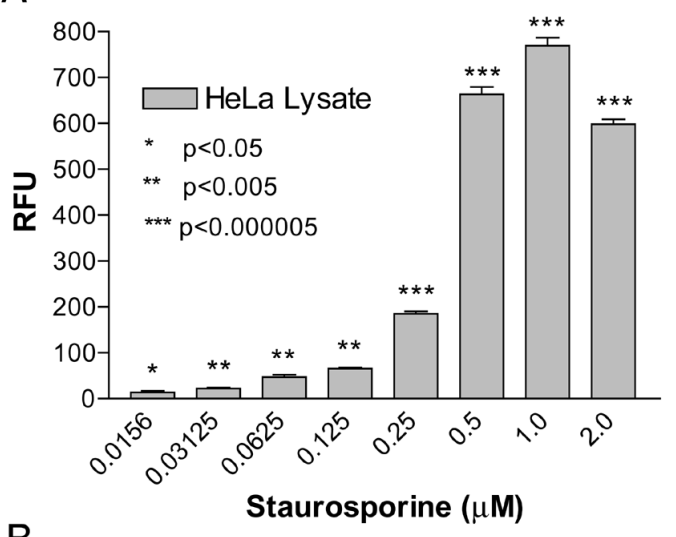

B

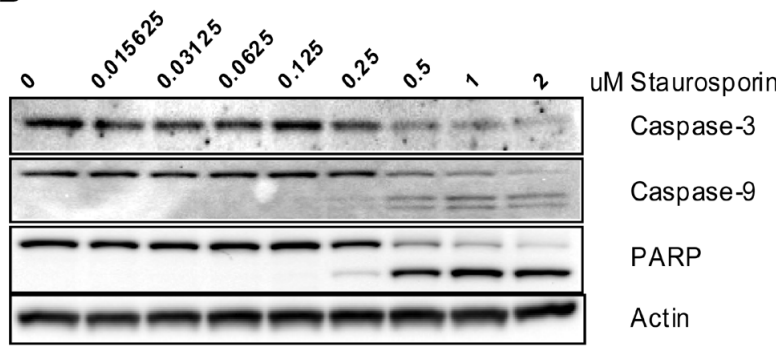

Figure 1. Induction of caspases-3/7 activity and PARP cleavage by staurosporine in HeLa cells. (A) HeLa cells $\left(2 \times 10^{6}\right)$ were allowed to adhere overnight in a $10-\mathrm{cm}$ Petri dish. The cells were treated with serially diluted staurosporine for $4 \mathrm{~h}$ to induce apoptosis. After the treatment period, the culture media were removed, the cells were washed twice with ice-cold PBS and lysed in the lysis buffer (20 mM Tris-Cl, pH 7.4, $5 \mathrm{mM}$ EDTA, $50 \mathrm{mM} \mathrm{NaCl}$, $10 \mathrm{mM}$ sodium pyrophosphate, $50 \mathrm{mM}$ sodium fluoride, and $1 \%$ Nonidet P40). Caspase- $3 / 7$ enzymatic assay was performed using $50 \mu \mathrm{g}$ of total protein (equating to 50000 cells) from each sample in an in vitro reaction performed in a 96-well plate. Fluoresence was measured with a CytoFluor fluorescence plate reader (excitation at $360 \mathrm{~nm}$ and emission at $460 \mathrm{~nm}$ ). Raw data (relative fluorescence unit, RFU), from which we subtracted the value of the untreated control, are plotted against the staurosporine concentration in the GraphPad Prism software. Statistical analysis was done using a Student's $t$ test. (B) Total protein ( $75 \mu \mathrm{g}$ for each sample) was electrophoretically separated on $4 \%-15 \%$ SDS-polyacrylamide gels and immunoblotted with antibodies against caspase-3, caspase-9, PARP, and actin control. compounds in a high-throughput format, we performed the assay on HeLa cells in a 96-well plate. As illustrated in Figure 2A, the assay is sensitive enough to detect caspase-3/7 activity in as few as 1000 cells/well $(P<$ $0.001)$. The caspase-3/7 activity was also highly significant $(P<0.000005)$ at $2000-50000$ cells/well. We found the assay to be as sensitive as the traditional method shown in Figure 1. Fig-

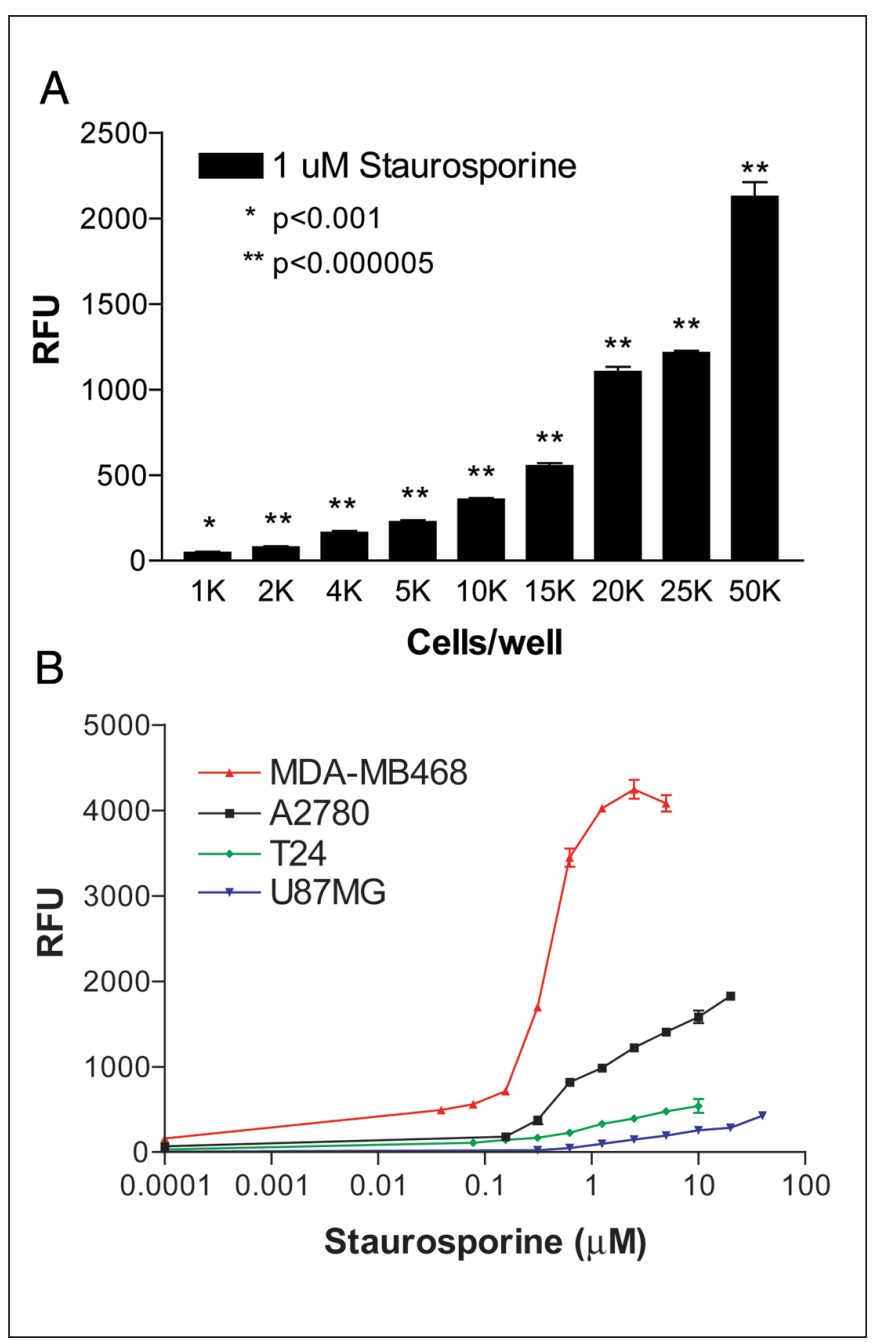

Figure 2. One-step caspase-3/7 assay. (A) HeLa cells were plated at 1000 $2000,4000,5000,10000,15000,20000,25000$, and 50000 cells/well in a 96-well plate. After overnight incubation, the cells were treated for $4 \mathrm{~h}$ with 1 $\mu \mathrm{M}$ staurosporine to induce apoptosis at $37^{\circ} \mathrm{C}$ in $5 \% \mathrm{CO}_{2}$. The control cells were treated with vehicle alone. The one-step caspase- $3 / 7$ assay buffer $(3 \times)$ was added to each well and incubated at $37^{\circ} \mathrm{C}$ in $5 \% \mathrm{CO}_{2}$ for $1 \mathrm{~h}$. Fluorescence was measured with a CytoFluor fluorescence plate reader (excitation at 360 $\mathrm{nm}$ and emission at $460 \mathrm{~nm}$ ). Raw data (RFU), from which we subtracted the value of the untreated control, are plotted against cell densities per well in the GraphPad Prism software. Statistical analysis was done using a Student's $t$ test. (B) The one-step caspase-3/7 assay was performed as described in the protocol on A2780, MDA-MB468, T24, and U87MG plated at 20000 cells/well in a 96-well plate. The cells were treated with serially diluted staurosporine for $4 \mathrm{~h}$ to induce apoptosis. 
ure $2 \mathrm{~B}$ shows that the assay is versatile enough to measure cellular caspase- $3 / 7$ enzymatic activity in multiple human tumor cell lines (A2780 ovarian carcinoma, MDA-MB468 breast carcinoma, T24 bladder carcinoma, and U87MG glioblastoma) treated with staurosporine, a potent kinase inhibitor known to induce apoptosis. The varying levels of caspase-3/7 activity observed in these cell lines are probably due to differences in the cell lines' sensitivity to staurosporine. Additionally, the sensitivity of the assay can be further increased by using substrates labeled with novel sensitive reagents.

In summary, the one-step cellular caspase- $3 / 7$ assay described here is a versatile, rapid, sensitive, and fairly inexpensive method that can be used in any research laboratory. The simplicity of the assay makes it flexible enough to be automated for high-throughput screening for drug discovery projects.

\section{REFERENCES}

1. Nicholson, D.W. 2000. From bench to clinic with apoptosis-based therapeutic agents. Nature 407:810-816.

2.Thornberry, N. and Y. Lazebnik. 1998. Caspases: enemies within. Science 281:13121316.

3.Yuan, J. 1997. Transducing signals of life and death. Curr. Opin. Cell Biol. 9:247-251.

4.Wyllie, A.H. 1997. Apoptosis: an overview. Brit. Med. Bull. 53:451-465.

5.Green, D.R. and J.C. Reed. 1998. Mitochondria and apoptosis. Science. 281:1309-1312.

6.Alnemri, E.S. 1997. Mammalian cell death proteases: a family of highly conserved aspartate specific cysteine proteases. J. Cell Biochem. 64:33-42.

7.Cryns, V. and Y. Yuan. 1998. Proteases to die for. Genes. Dev. 12:1551-1570.

8.Salvesen, G.S. and V.M. Dixit. 1999. Caspase activation: the induced-proximity model. Proc. Natl. Acad. Sci. USA 96:10964-10967.

9.Salvesen, G.S. and V.M. Dixit. 1997. Caspases: intracellular signaling by proteolysis. Cell 91:443-446.

10.Villa, P., S.H. Kaufmann, and W.C. Earnshaw. 1997. Caspases and caspase inhibitors. Trends Biochem. Sci. 22:381-393.
11.Reed, J. 1998. Caspases and cytokines: roles in inflammation and autoimmunity. Adv. Immunol. 73:265-287.

12.Thompson, C.B. 1995. Apoptosis in the pathogensis and treatment of the disease. Science. 267:1456-1462.

13.Miller, L.J. and J. Marx. 1998. Apoptosis. Science 281:1301.

Received 13 December 2002; accepted 13 March 2003.

\section{Address correspondence to:}

Dr. Bharvin K.R. Patel

Division of Cancer Research

Eli Lilly and Company

Lilly Corporate Center

Indianapolis, IN 46285-0424, USA

e-mail:patel_bharvin@lilly.com 